\title{
Vaccine against PCSK9: the natural strategy from passive to active immunization for the prevention of atherosclerosis
}

\author{
Fernando Civeira, Estíbaliz Jarauta \\ Hospital Universitario Miguel Servet, IIS Aragon, CIBERCV, Universidad de Zaragoza, Zaragoza, Spain \\ Correspondence to: Dr. Fernando Civeira, MD, PhD. Hospital Universitario Miguel Servet, Avda Isabel La Católica 1-3, 50009 Zaragoza, Spain. \\ Email: civeira@unizar.es. \\ Provenance: This is an invited Editorial commissioned by the Section Editor Hai-Long Dai (Department of Cardiology, Yan'an Affiliated Hospital of \\ Kunming Medical University, Kunming, China). \\ Comment on: Landlinger C, Pouwer MG, Juno C, et al. The AT04A vaccine against proprotein convertase subtilisin/kexin type 9 reduces total \\ cholesterol, vascular inflammation, and atherosclerosis in APOE*3Leiden.CETP mice. Eur Heart J 2017;38:2499-507.
}

Submitted Oct 03, 2017. Accepted for publication Oct 09, 2017.

doi: $10.21037 /$ jtd.2017.10.18

View this article at: http://dx.doi.org/10.21037/jtd.2017.10.18

Chronic diseases in general, and those derived from atherosclerosis in particular, are the major health challenge today in both developed and developing countries (1). In the case of atherosclerosis is a paradox because we know the mechanisms that produce the disease and we have effective tools against those mechanisms, but for different reasons we cannot control in many cases the consequences of arterial plaques. The hopes at the end of the last century on the disappearance of most diseases related to atherosclerosis in the short term have been diluted, and these hopeful expectations do not seem to be fulfilled in the coming years $(2,3)$.

Any strategy for the reduction of cardiovascular diseases should have as main objective the primary prevention of the disease and to reach this goal, we must have a clear strategy based on the prevention of the appearance of its risk factors and on their early treatment if present. Currently, most health efforts are focused on the treatment of subjects with clinical disease or with a high short-term risk of developing it (4), and this strategy, although effective in some cases, signifies a not acceptable high residual disease and costs (5).

Among the most effective strategies in cardiovascular prevention is the reduction of cholesterol transported in low density lipoproteins (LDL). Evidence for this approach is incontrovertible coming from multiple epidemiology, genetic and intervention studies. Furthermore, the studies of Mendelian randomization with genetic variants that reduce LDL cholesterol show us the enormous impact that population reductions of LDL cholesterol would have on the reduction of cardiovascular diseases if these reductions were applied to large population groups from the early ages of life (6).

At present, the reduction of LDL cholesterol is based on dietary and life style measures that basically consist of maintaining an ideal weight and reducing the intake of saturated fatty acids and trans fatty acids. As second step, the use of drugs is recommended, where statins occupy a very preferential place, but only in cases of high risk in the short term or very high concentration of LDL cholesterol (4). However, this strategy has many weaknesses. The results of clinical trials based on dietary changes, with rare exceptions such as the PREDIMED study with olive oil and nuts (7), are disappointing and it does not appear that this strategy, although necessary, will substantially modify the disease in the coming years (8). The second option, pharmacological treatment, has many limitations and drawbacks. Statins have side effects that limit their use in a non-negligible percentage of patients, require a daily administration over the years that leads to a huge therapeutic failure over time, taking drugs throughout life is a practice badly accepted by many fellow citizens, and the medication prices, will not be acceptable for certain economies (9). Moreover, although age-standardized mortality from atherosclerosis has diminished in many areas, the absolute number of cardiovascular deaths associated with atherosclerosis continues to increase, with the majority now occurring in middle- and low-income countries (3). 
It becomes necessary new strategies in reducing LDL cholesterol that solvents all these problems. Recently, Landlinger et al. have published the results of a cholesterollowering vaccine that safely decrease total cholesterol, vascular inflammation, and atherosclerosis (10). They use a peptide-based vaccine directed against proprotein convertase subtilisin/kexin type 9 (PCSK9) called AT04A in the $\mathrm{APOE}^{*} 3$ Leiden CETP transgenic mice. a mouse model prone to the development of arteriosclerosis. The human and homolog mouse PCSK9 protein (153aa692aa) was formulated into the AT04A vaccine. Mice were immunized five times subcutaneously either with AT04A or a control vaccine. Four weeks after prime immunization normal chow was switched to a hypercholesterolemic diet in order to induce atherosclerosis. AT04A induced a strong and persistent immune response, which reached a maximum 12 weeks after immunization. AT04A vaccine treated mice showed a highly significant decrease in PCSK9 concentration up to 59\%, plasma total cholesterol was decreased by $53 \%$, and a significantly reduced lesion area (-64\%, compared with control animals). Moreover, AT04A treated animals showed significative reductions in the arterial necrotic core areas of $77 \%$ and reductions in inflammatory biomarkers such as serum amyloid A, macrophage-derived chemokine (MDC/ CCL22), and vascular endothelial growth factor A (VEGF-A). Landlinger's study is the first to show that active immunization against PCSK9 does not only reduces total cholesterol, but also vascular inflammation and atherosclerotic development in a mouse model of dietinduced atherosclerosis.

Vaccination against infectious diseases has been one the most efficient therapeutic procedures in medical history. The prophylactic induction of antibodies against different pathogen antigens has proven to be highly effective in the prevention of multiple bacterial and viral infections. Furthermore, vaccination is a safe and well-accepted approach for the public and health systems, is easy to apply to large proportions of the population in almost any country, with affordable costs, and, more importantly, preventing rather than treating a specific disease, so many complications, patient suffering and disease-related costs can be avoided (11). An effective prophylactic vaccination against atherosclerosis would a breakthrough in medicine.

Numerous components of the innate and adaptive immune systems participate in modulating atherogenesis, so multiple vaccines have been attempted in the last decades (12). The research on human vaccination against atherosclerosis can be classified according to two different approaches: the first one is to modulate immune responses to increase protective immunity that, spontaneously, is not potent enough, or to decrease deleterious immune response favoring the development of the disease. Vaccinations against oxLDL epitopes and heat shock protein 60 (HSP 60) are models to increase pre-existing immune responses considered part of the pathologic process that have demonstrated to protect against atherosclerosis in mice. In the other side of the balance is the immunization against mediators of inflammation involved in the development of the atherosclerosis lesions such as proinflammatory $\mathrm{CD}^{+}$Th cells or certain cytokines such as IL1 $\beta$. Many of these immunizations have demonstrated some benefit in animal models of atherosclerosis (13).

The second approach is to produce neutralizing antibodies that inhibit the effect of a proatherogenic protein that is chosen as antigen. Immunization with peptides derived from apolipoprotein B-100, the main protein of LDL particles or against cholesterol ester transfer protein (CETP) involved in the transfer of esterified cholesterol from HDL to apolipoprotein B-containing lipoproteins also significantly reduced the development of atherosclerosis (14). In the Landlinger's work, mentioned above, PCSK9 was chosen as antigen. PCSK9 plays a fundamental role in LDL metabolism because binds to the LDL receptor in the cell surface and facilitates the degradation of the LDL receptor. Consequently, the reduction of PCSK9 activity is associated with increased LDL particle uptake by their receptors and reduction in LDL cholesterol concentration in blood (15). Moreover, gain of function mutations in PCSK9 produced familial hypercholesterolemia (16), and loss of function genetic variation in PCSK9 are associated with low LDL cholesterol and reduced cardiovascular risk (17). Recently, two large clinical trials with evolocumab and bococizumab, two different human PCSK9 monoclonal antibodies, have demonstrated the clinical benefit of passive immunization against PCSK9 on top of statins in subjects very high cardiovascular risk without any relevant side effect associated with treatment $(18,19)$. However, the costs of monoclonals and their inconvenience for the patient could be major impediments for their extensive use. Therefore, all these data support PCSK9 protein as an excellent candidate for active immunization with a vaccine moving from passive administration to active vaccination.

Safety in addition to efficacy is a key issue in order to develop a successful vaccine against atherosclerosis (12). Therapy with monoclonal antibodies does not involve 
active engagement of the host's immune system that could associate non- desirable side effects. The safety of vaccination against a self-molecule, such as PCSK9, would result in reduced plasma concentrations of a natural protein with key function in the hepatic LDL receptors half-life, but it is expressed in the liver and also in other tissues and may have other important physiological functions than can be altered by a sustained reduction with active immunization (20). Non-LDL cholesterol potential functions of PCSK9 have been recently reviewed by Banerjee $e t a l$. and include: hepatic regeneration; pancreatic integrity; glucose homeostasis; antiviral activity; antimalarial activity; regulation of different cell signaling pathways; cortical neural differentiation; and neuronal apoptosis (20). At present, data from homozygous subjects for PCSK9 null alleles with absence of PCSK9 from birth (21), and from large clinical trial with monoclonal antibodies against PCSK9 do not show any dangerous signal $(18,19)$, but continuous monitoring of these populations in coming years will solve this important issue. Furthermore, as PCSK9 is a cell surface molecule, an active immunization has a potential antibody-dependent cell-mediated cytotoxicity or complement-dependent cell lysis with nonspecific destruction of these cells (13). To improve safety it would be necessary, at least initially, that the immune response would be reversible and the factors that govern the duration of antibody should be taken in consideration. Probably, short protein-based vaccines without adjuvants seem to be the most likely candidates for the induction of reversible antibody responses that would also limit unwanted T-cell responses (22).

If target-related safety questions are solved and the longterm safety data with monoclonals is good then therapeutic vaccination against PCSK9 will be a breakthrough to prevent and treat atherosclerosis. The simplicity and favorable cost of vaccines in comparison of monoclonal antibodies would result in better acceptance of patients and payers, even in the poorest countries. So, Landlinger's work is an enormous step ahead in atherosclerosis prevention. Phase I clinical trial are underway with their PCSK9 vaccine and the scientific community will be eager to know the safety of this treatment. PCSK9 inhibition has created many expectations. We already have monoclonal antibodies that improve cardiovascular disease, it is feasible than in several years a successful vaccine could be offered to a large spectrum of subjects worldwide. If this approach results in cardiovascular disease reduction, then it will be time to inactivate the PCSK9 gene with a technique such as
CRISPR Cas9 (23). The future looks exiting.

\section{Acknowledgements}

Funding: This work was funded by the Spanish Ministry of Health FIS PI15/01983, and CIBERCV (Supported with European grants).

\section{Footnote}

Conflicts of interest: F Civeira received lectures and advisory fees from Sanofi Aventis, Amgen, Pfizer, Merck and Ferrer. The other author has no conflicts of interest to declare.

\section{References}

1. Bachmann MF, Dyer MR. Therapeutic vaccination for chronic diseases: a new class of drugs in sight. Nat Rev Drug Discov 2004;3:81-8.

2. Brown MS, Goldstein JL. Heart attacks: gone with the century? Science 1996;272:629.

3. Joseph P, Leong D, McKee M, et al. Reducing the Global Burden of Cardiovascular Disease, Part 1: The Epidemiology and Risk Factors. Circ Res 2017;121:677-94.

4. Authors/Task Force Members, Catapano AL, Graham I, et al. 2016 ESC/EAS Guidelines for the Management of Dyslipidaemias: The Task Force for the Management of Dyslipidaemias of the European Society of Cardiology (ESC) and European Atherosclerosis Society (EAS) Developed with the special contribution of the European Assocciation for Cardiovascular Prevention \& Rehabilitation (EACPR). Atherosclerosis 2016;253:281-344.

5. Noto D, Cefalù AB, Averna MR. Beyond statins: new lipid lowering strategies to reduce cardiovascular risk. Curr Atheroscler Rep 2014;16:414.

6. Ference BA, Ginsberg HN, Graham I, et al. Low-density lipoproteins cause atherosclerotic cardiovascular disease. 1. Evidence from genetic, epidemiologic, and clinical studies. A consensus statement from the European Atherosclerosis Society Consensus Panel. Eur Heart J 2017;38:2459-72.

7. Estruch R, Ros E, Salas-Salvadó J, et al. Primary prevention of cardiovascular disease with a Mediterranean diet. N Engl J Med 2013;368:1279-90.

8. Look AHEAD Research Group, Wing RR, Bolin P, et al. Cardiovascular effects of intensive lifestyle intervention in type 2 diabetes. N Engl J Med 2013;369:145-54. 
9. Maningat P, Gordon BR, Breslow JL. How do we improve patient compliance and adherence to long-term statin therapy? Curr Atheroscler Rep 2013;15:291.

10. Landlinger C, Pouwer MG, Juno C, et al. The AT04A vaccine against proprotein convertase subtilisin/kexin type 9 reduces total cholesterol, vascular inflammation, and atherosclerosis in APOE*3Leiden.CETP mice. Eur Heart J 2017;38:2499-507.

11. Ummarino D. Dyslipidaemia: Anti-PCSK9 vaccines to halt atherosclerosis. Nat Rev Cardiol 2017;14:442-3.

12. Govea-Alonso DO, Beltrán-López J, Salazar-González JA, et al. Progress and future opportunities in the development of vaccines against atherosclerosis. Expert Rev Vaccines 2017;16:337-50.

13. Shah PK, Chyu KY, Dimayuga PC, et al. Vaccine for atherosclerosis. J Am Coll Cardiol 2014;64:2779-91.

14. Fredrikson GN, Söderberg I, Lindholm M, et al. Inhibition of atherosclerosis in apoE-null mice by immunization with apoB-100 peptide sequences. Arterioscler Thromb Vasc Biol 2003;23:879-84.

15. Qian YW, Schmidt RJ, Zhang Y, et al. Secreted PCSK9 downregulates low density lipoprotein receptor through receptor-mediated endocytosis. J Lipid Res 2007;48:1488-98.

16. Abifadel M, Varret M, Rabès JP, et al. Mutations in

Cite this article as: Civeira F, Jarauta E. Vaccine against PCSK9: the natural strategy from passive to active immunization for the prevention of atherosclerosis. J Thorac Dis 2017;9(11):4291-4294. doi: 10.21037/jtd.2017.10.18
PCSK9 cause autosomal dominant hypercholesterolemia. Nat Genet 2003;34:154-6.

17. Cohen JC, Boerwinkle E, Mosley TH Jr, et al. Sequence variations in PCSK9, low LDL, and protection against coronary heart disease. N Engl J Med 2006;354:1264-72.

18. Ridker PM, Revkin J, Amarenco P, et al. Cardiovascular Efficacy and Safety of Bococizumab in High-Risk Patients. N Engl J Med 2017;376:1527-39.

19. Sabatine MS, Giugliano RP, Keech AC, et al. Evolocumab and Clinical Outcomes in Patients with Cardiovascular Disease. N Engl J Med 2017;376:1713-22.

20. Banerjee Y, Santos RD, Al-Rasadi K, et al. Targeting PCSK9 for therapeutic gains: Have we addressed all the concerns? Atherosclerosis 2016;248:62-75.

21. Abifadel M, Elbitar S, El Khoury P, et al. Living the PCSK9 adventure: from the identification of a new gene in familial hypercholesterolemia towards a potential new class of anticholesterol drugs. Curr Atheroscler Rep 2014;16:439.

22. Bachmann MF, Whitehead P. Active immunotherapy for chronic diseases. Vaccine 2013;31:1777-84.

23. Nelson CE, Robinson-Hamm JN, Gersbach CA. Genome engineering: a new approach to gene therapy for neuromuscular disorders. Nat Rev Neurol 2017 [Epub ahead of print]. 